\title{
Uso de membrana de celulosa oxidada post-gingivectomía en hiperplasia gingival inducida por fenitoína.
}

\author{
Use of oxidized cellulose regenerated post-gingivectomy \\ on ginigival hyperplasia induced by phenytoin.
}

\author{
Luis Eduardo Poblano-Vázquez,* Elsa Patricia Furrer-Franco,* Gilberto Zataraín-Hernández,* \\ Lizeth Alejandra Reyes-Alvarado*
}

\section{RESUMEN}

Introducción: La hiperplasia gingival es una condición benigna caracterizada por el aumento de volumen de la encía. Algunos fármacos, factores genéticos, aparatología y placa dentobacteriana son factores que pueden inducir esta condición. Objetivo: Devolver la anatomía a la encía brindando una mejor estética y permitiendo una óptima higiene oral. Material y métodos: Paciente masculino de 20 años de edad con antecedentes de fenitoína presenta aumento de volumen en la encía. Resultados: Se obtuvieron resultados estéticos y funcionales satisfactorios con el tratamiento quirúrgico y el uso de membrana de celulosa oxidada. Conclusión: En el manejo de la hiperplasia gingival es importante el enfoque no quirúrgico como control de placa dentobacteriana y medidas de higiene del mismo paciente.

Palabras clave: Hiperplasia gingival, fenitoína, celulosa oxidada.

\section{ABSTRACT}

Introduction: Gingival hyperplasia is a benign condition characterized for the grown on the gingival volume. Some drugs, genetic, orthodontic and dental plaque are some factors that can induce this condition. Objective: To return the gingival anatomy, providing a better aesthetic allowing also good oral hygiene. Material and methods: A male 20 years of age with medical history of phenytoin display grown on the gingival volume. Results: Aesthetic and functional results were achieved with the surgical treatment and the oxidized cellulose membrane. Conclusion: In the gingival hyperplasia management is important de non-surgical approach, as dental plaque control and oral hygiene of the patient.

Keywords: Gingival hyperplasia, phenytoin, oxidized cellulose.

\section{INTRODUCCIÓN}

$\mathrm{L}$ a hiperplasia gingival, también denominada como fibromatosis gingival o agrandamiento gingival, ${ }^{1}$ es una condición benigna de la cavidad oral, la cual inicia en la papila interdental como un agrandamiento nodular y puede ir creciendo en dirección coronal alterando la forma y el margen gingival. ${ }^{2,3}$ Es un efecto secundario o consecuencia del consumo de medicamentos como anticonvulsionantes, inmunosupresores y bloqueadores de calcio; ; ${ }^{4,5}$ sin embargo, el agrandamiento gingival es una de las condiciones que se presenta con más frecuencia en pacientes con tratamiento de ortodoncia. ${ }^{6}$ Las neoplasias, alteraciones hormonales (embarazo, menopausia), herencia y discrasias sanguíneas son otros factores que pueden inducir el crecimiento gingival. ${ }^{1,7}$

Múltiples medicamentos están asociados a hiperplasia gingival, ${ }^{4}$ siendo su incidencia total de $50 \% .{ }^{8}$ La incidencia de esta condición en pacientes que toman ciclosporina A es de 20 a $80 \%,{ }^{9}$ de $20 \%$ en pacientes que ingieren nifedipino, ${ }^{2}$ y de 13 a $50 \%$ en pacientes con fenitoína como tratamiento. ${ }^{10}$

\footnotetext{
* Cirujano Dentista. Maestría en Ciencias Odontológicas con acentuación en Periodoncia de la Facultad de Odontología, unidad Torreón, Universidad Autónoma de Coahuila. México.

Recibido: 25 de junio de 2020. Aceptado: 11 de enero de 2022.

Citar como: Poblano-Vázquez LE, Furrer-Franco EP, Zataraín-Hernández G, Reyes-Alvarado LA. Uso de membrana de celulosa oxidada post-gingivectomía en hiperplasia gingival inducida por fenitoína. Rev ADM. 2022; 79 (1): 54-58. https://dx.doi.org/10.35366/103819
}

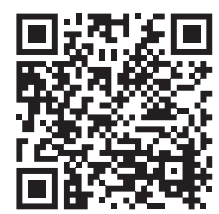


Los primeros reportes acerca de los efectos secundarios en los tejidos gingivales inducidos por fenitoína datan de 1939 por Kimbal. ${ }^{11}$ El sexo, la edad y el uso de dosis altas y/o consumo prolongado del medicamento son factores del desarrollo de crecimiento gingival, manifestándose entre el primer y tercer mes. 2,10,12

Las características clínicas y síntomas de esta patología incluyen inflamación, sangrado, sensibilidad y movimiento dental anormal, llevando a tener consecuencias como maloclusión, cambios estéticos, problemas con la masticación y pronunciación, y formación y desarrollo de caries y enfermedades periodontales debido a la dificultad en el mantenimiento de la higiene oral. $3,4,10$

Se cree que estos medicamentos alteran las hormonas como la testosterona, causando una proliferación celular en la encía. ${ }^{13}$ Histológicamente el agrandamiento gingival inducido por medicamentos se caracteriza por el aumento de los componentes de la matriz extracelular, principalmente el colágeno. ${ }^{1,2}$ No todos los fármacos inducirán el agrandamiento gingival de la misma manera histológicamente, las lesiones más fibróticas son provocadas por la fenitoína, mientras que las menos fibróticas son desarrolladas por la ciclosporina, pero con mayor concentración de contenido inflamatorio, y el nifedipino producirá lesiones de contenido mixto. ${ }^{8}$

La terapia no quirúrgica consiste en la prevención enfocada en los factores predisponentes, principalmente en la disminución de carga bacteriana por medio de detartajes, que puede reducir hasta $40 \%$ la lesión, también el uso de enjuagues de clorhexidina ha demostrado reducción del crecimiento gingival. ${ }^{8,14,15} \mathrm{El}$ uso de la azitromicina ha sido estudiado por sus efectos en el metabolismo del colágeno para tratar el agrandamiento gingival. ${ }^{16} \mathrm{El}$ ajuste

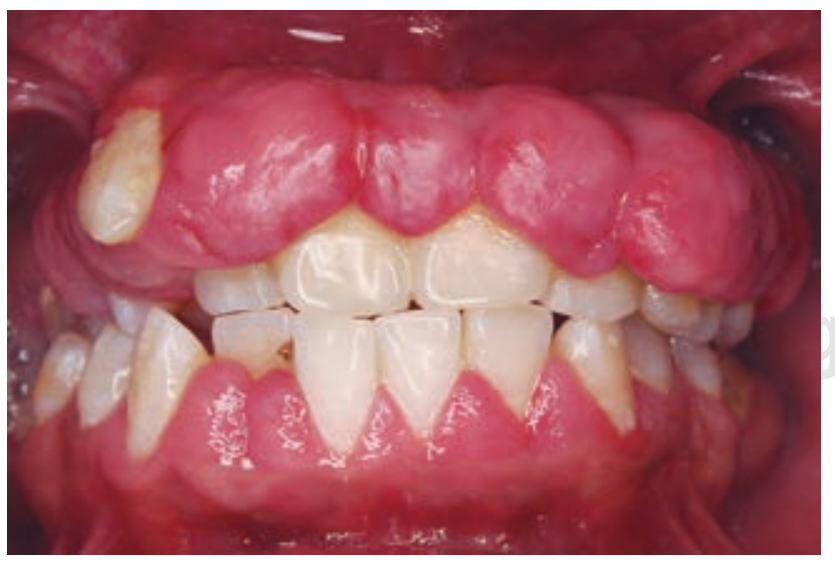

Figura 1: Foto frontal inicial.

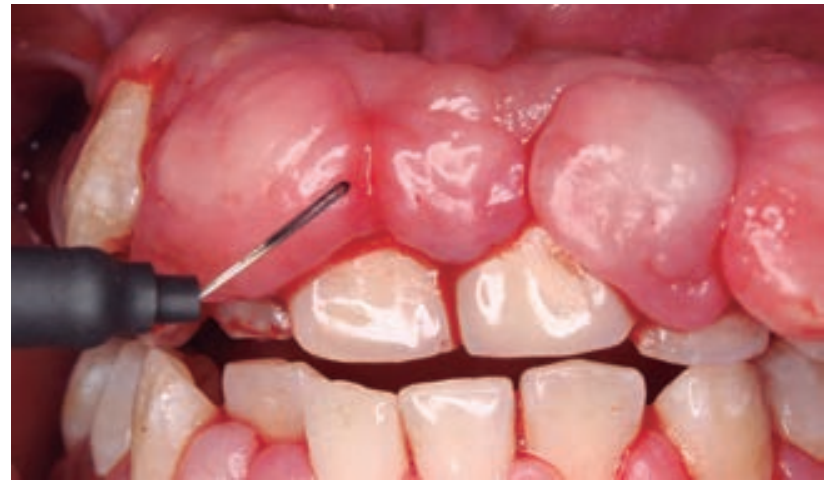

Figura 2: Gingivectomía con electrobisturí.

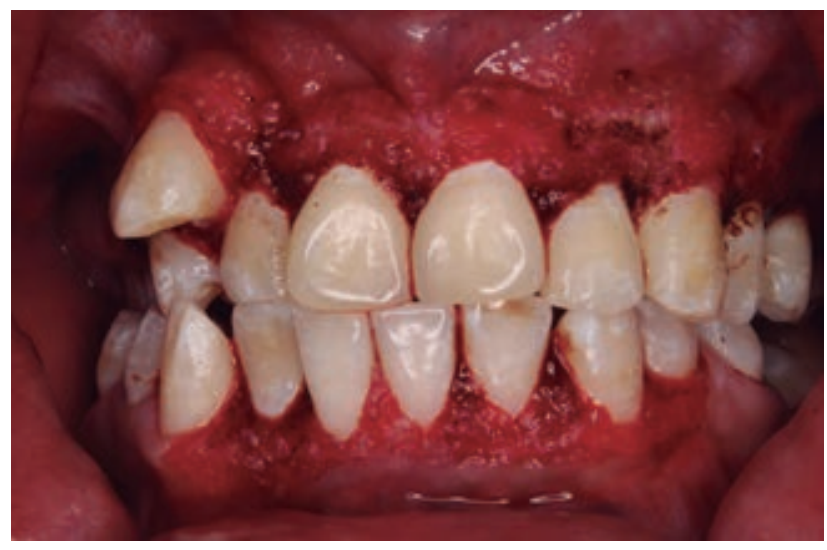

Figura 3: Foto de gingivectomía final.

de dosis o cambio de medicamento es otra opción para el manejo del padecimiento; sin embargo, en ciertos casos esta opción no es viable y hay que intervenir quirúrgicamente para la escisión de la lesión, ${ }^{7}$ teniendo menor índice de reincidencia cuando se lleva a cabo con láser. ${ }^{17}$

La cicatrización y regeneración de tejidos va a pasar por cuatro etapas: hemostasia, inflamación, proliferación y remodelación; ${ }^{18}$ estas heridas tendrán que sobrepasar algunos obstáculos como la epitelización tardía, sangrado persistente y drenaje prolongado, al mismo tiempo existirán factores que pueden retrasar la cicatrización, entre ellos la edad avanzada, diabetes no controlada, obesidad y enfermedades vasculares. ${ }^{19}$

Además de su efecto hemostático y mínima reacción al tejido, los agentes hemostáticos también deben ser biodegradables, económicos y no antigénicos. ${ }^{20}$ La celulosa oxidada regenerada es un agente hemostático utilizado por décadas desde $1943,{ }^{20,21}$ es una matriz derivada 
de la celulosa que absorberá el exudado y formará una protección en la herida, su bajo $\mathrm{pH}$ brinda un potencial antimicrobiano y al mismo tiempo un efecto hemostático formador de un coágulo ficticio, por lo cual es una buena opción para el tratamiento en heridas. ${ }^{19,22}$ La celulosa oxidada regenerada va a interactuar en la activación de la cascada de coagulación, plaquetas y proteínas, teniendo así su acción como hemostático. ${ }^{20}$

El objetivo de este estudio es evaluar los beneficios que brinda la membrana de celulosa oxidada al aplicarla en sitio postquirúrgico en un paciente con hiperplasia gingival inducida por fenitoína.

\section{PRESENTACIÓN DEL CASO}

Paciente masculino de 20 años de edad asiste a consulta a la Clínica del Postgrado de Periodoncia en la Facultad de Odontología de la Universidad Autónoma de Coahuila, siendo el motivo de consulta «inconformidad estética y dificultad para masticar debido al crecimiento de encías». Se realizó una anamnesis indirecta por medio de la madre, refiriéndonos que surgió una complicación durante el parto, dejando como secuelas episodios de catalepsia. La tutora del paciente refiere llevar 10 años con fenitoína como tratamiento para dichos episodios, haciendo hincapié que desde entonces empezó a notar el aumento de encía.

Al realizar el examen clínico se observó presencia generalizada de placa dentobacteriana y malposición dental, aumento de volumen en la encía en las caras vestibulares y palatinas del sextante \#2, y en la cara vestibular del sextante \#5 de color rosa pálido con márgenes eritematosos y nodulares cubriendo un tercio de la corona clínica, al momento de sondear hubo presencia de sangrado (Figura 1).

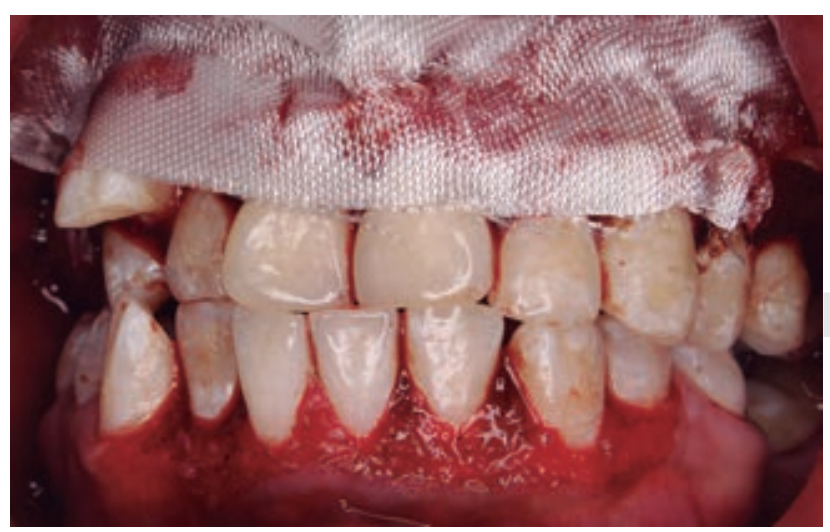

Figura 4: Colocación de membrana de celulosa.

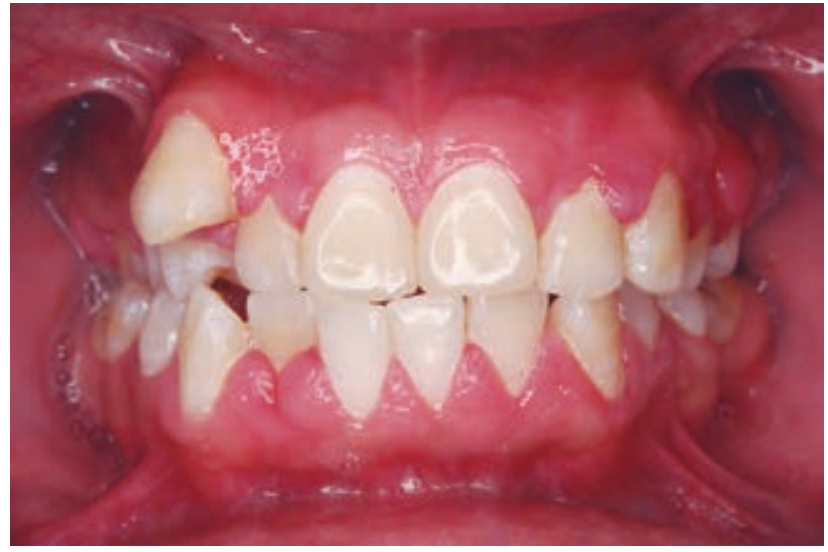

Figura 5: Cinco meses de evolución. Se observa acúmulo de placa y aumento de volumen en papilas.

El procedimiento quirúrgico se llevó a cabo bajo sedación y anestesia local. Se inició el detartaje con ultrasonido para disminuir la carga bacteriana en la misma cita debido a la poca cooperación del paciente. Se inició la gingivectomía con electrobisturí y se continuó con hoja de bisturí \#15 y tijeras LaGrange (Figura 2).

Se realizó la gingivectomía y gingivoplastia en ambos sextantes (Figura 3). Terminando el procedimiento quirúrgico se colocó una membrana de satín hemostático absorbible (Lides S-100) en la cara vestibular del sextante \#2 (Figura 4). Se recetó amoxicilina de 500 mg cada ocho horas por siete días, ibuprofeno $600 \mathrm{mg}$ cada ocho horas por cinco días, ketorolaco $10 \mathrm{mg}$ cada ocho horas por cinco días, y enjuagues de clorhexidina al $12 \%$ con indicaciones al paciente y se le dieron citas de revisión (Figura 5).

\section{RESULTADOS}

Al colocar la membrana de celulosa oxidada regenerada (Lides S-100) se logró menor grado de inflamación y mayor protección en el sitio quirúrgico. El paciente no refiere dolor ni inconformidad postquirúrgica. A los 15 días de evolución se observa el tejido gingival un poco eritematoso ya epitelizado, se decide realizar una segunda profilaxis para retirar restos de placa dentobacteriana. Dos meses después del procedimiento quirúrgico, el paciente presenta conformidad con la estética, se observa el tejido completamente epitelizado y color rosa pálido, encontrándose leve acúmulo de placa en zona anterior, se le refuerza al paciente y a su tutora la técnica de cepillado. A los cinco meses de evolución se 
observa buena cicatrización; sin embargo, hay presencia de papilas con aumento breve de volumen y eritematosas relacionado al acúmulo de placa dental y al uso continuo de la fenitoína.

\section{DISCUSIÓN}

La hiperplasia gingival se caracteriza por un aumento de volumen de la encía pudiendo ser inducida por ciertos medicamentos. ${ }^{2}$ El primer enfoque dado a esta condición se basa en la terapia no quirúrgica, incluyendo eliminación de placa dental y/o modificación de dosis o tipo de medicamento. ${ }^{14,15}$ El enfoque quirúrgico se basará en la reducción del volumen gingival por medio del recorte de encía. ${ }^{7}$

El uso del electrobisturí nos brindará ventajas que el bisturí no tiene, entre ellas, una cirugía libre de sangrado, movimientos sutiles y de fácil aprendizaje, un postoperatorio menos molesto y una mínima formación de cicatriz. ${ }^{23}$

Se han propuesto diferentes tipos de hemostáticos para heridas o lesiones en los tejidos del cuerpo como músculo, mucosa y piel. ${ }^{18}$ Entre los hemostáticos más utilizados podemos encontrar la celulosa oxidada regenerada, cera para hueso, colágeno, esponja de gelatina, entre otros. ${ }^{22}$ Aparte del efecto hemostático, se busca entre estos agentes propiedades cicatrizantes (habiendo una mejor o más pronta epitelización), disminución del dolor postquirúrgico y del riesgo de infección. ${ }^{19}$

En este artículo se presenta un caso de hiperplasia gingival generalizada inducida por medicamentos. Debido a la gran extensión de esta condición, se llevará a cabo un procedimiento quirúrgico combinando el uso de electrobisturí por sus propiedades y el uso de bisturí y otros instrumentos cortantes para el remodelado. De igual manera, se decidió el manejo de una membrana de celulosa oxidadareal regenerada (Lides S-100) para evitar complicaciones postquirúrgicas como dolor, sangrado o infecciones.

\section{CONCLUSIONES}

El manejo de la hiperplasia gingival inducida por medicamentos debe enfocarse primero en la terapia no quirúrgica, tratando los factores que inducen y exacerban esta condición como el control de placa, modificación de medicamento o dosis, materiales utilizados en ortodoncia; sin embargo, no todos los factores pueden ser modificables como los factores genéticos, hormonales o fármacos que no pueden ser cambiados.
Cuando el manejo no quirúrgico del crecimiento gingival se lleva a cabo (profilaxis, modificación de medicamento) y la encía sigue teniendo un aumento de volumen, ésta puede conllevar a comprometer la estética y la higiene oral, al igual que en otros procedimientos como la aparatología ortodóntica o prótesis dentales. Cuando es así, se continúa con el enfoque quirúrgico para disminuir el volumen gingival y regresarlo a su anatomía normal o ideal. Existen diferentes instrumentos y aparatos para el recorte gingival, pudiéndose utilizar hoja y mango de bisturí, electrobisturí, tijeras LaGrange, entre otros, teniendo como finalidad la disminución y remodelación de la anatomía gingival.

Los procedimientos quirúrgicos extensos o muy invasivos producen incomodidades al paciente y son más propensos a conllevar sangrados. Se han propuesto múltiples hemostáticos para el manejo hemostático en heridas extensas, entre ellos, la celulosa oxidada regenerada. Ésta se encuentra en diferentes presentaciones (gasa, membrana), su $\mathrm{pH}$ bajo proporciona beneficios hemostáticos, además de brindar una protección al sitio quirúrgico dando mayor comodidad al paciente y previniendo así complicaciones postquirúrgicas como sangrado, infecciones y dolor, lo que la hace ideal para este tipo de tratamientos donde se deja el tejido desepitelizado expuesto.

\section{REFERENCIAS}

1. El-Menoufy H, Aziz Aly LA, Ragae A. Collagen turnover induced by cellular connective tissue cytokines of drug induced gingival overgrowth and hereditary gingival fibromatosis (histological and immunohistochemical comparative study). Future Dental Journal. 2016; 2 (1): 28-36

2. Manzur-Villalobos I, Díaz-Rengifo IA, Manzur-Villalobos D, DíazCaballero AJ. Agrandamiento gingival farmacoinducido: serie de casos. Univ Salud. 2018; 20 (1): 89-96.

3. Straka M, Varga I, Erdelsky I, Straka-Trapezanlidis M, Krnoulová J. Drug-induced gingival enlargement. Neuro Endocrinol Lett. 2014; 35 (7): 567-576.

4. Hatahira H, Abe J, Hane Y, Matsui T, Sasaoka S, Motooka Y et al. Drug-induced gingival hyperplasia: a retrospective study using spontaneous reporting system databases. J Pharm Health Care Sci. 2017; 3: 19.

5. Chang CW, Yang CJ, Lai YL. Phenytoin- and amlodipine-induced gingival overgrowth. J Dent Sci. 2012; 7 (1): 85-88.

6. Gómez Arcila V, Mercado Camargo J, Herrera Herrera A, Fang Mercado L, Díaz Caballero A. Níquel en cavidad oral de individuos con agrandamiento gingival inducido por tratamiento ortodóncico. Rev Clin Periodoncia Implantol Rehabil Oral. 2014; 7 (3): 136-141.

7. Ocaña Diestra T, García Linares S. Agrandamiento gingival por bloqueantes de canales de calcio. Odontol Sanmarquina. 2014; 17 (1): 12-15

8. Trackman PC, Kantarci A. Molecular and clinical aspects of druginduced gingival overgrowth. J Dent Res. 2015; 94 (4): 540-546. 
9. Lauritano D, Palmieri A, Lucchese A, Di Stasio D, Moreo G, Carinci F. Role of cyclosporine in gingival hyperplasia: an in vitro study on gingival fibroblasts. Int J Mol Sci. 2020; 21 (2): 595.

10. Candotto V, Pezzetti F, Baj A, Beltramini G, Lauritano D, Di Girolamo $M$ et al. Phenytoin and gingival mucosa: A molecular investigation. Int J Immunopathol Pharmacol. 2019; 33: 2058738419828259.

11. Suneja B, Chopra S, Thomas AM, Pandian J. A clinical evaluation of gingival overgrowth in children on antiepileptic drug therapy. J Clin Diagn Res. 2016; 10 (1): ZC32-ZC36.

12. Joshi NH, Deshpande AN, Deshpande NC, Rathore AS. Comparative evaluation of oral hygiene status and gingival enlargement among epileptic and healthy children as related to various antiepileptic drugs. J Indian Soc Periodontol. 2017; 21 (2): 125-129.

13. George A, George SP, John S, George N, Joe S, Mathew R. Changes in inflammatory markers in bacterial- and nifedipine-induced gingival inflammation. J Int Oral Health. 2015; 7 (Suppl 2): 64-67.

14. Pejcic A, Djordjevic V, Kojovic D, Zivkovic V, Minic I, Mirkovic D et al. Effect of periodontal treatment in renal transplant recipients. Med Princ Pract. 2014; 23 (2): 149-153.

15. Gau CH, Tu HP, Chin YT, Chen RY, Fu MM, Fu E. Can chlorhexidine mouthwash twice daily ameliorate cyclosporine-induced gingival overgrowth? J Formos Med Assoc. 2013; 112 (3): 131-137.

16. Paik JW, Kim CS, Cho KS, Chai JK, Kim CK, Choi SH. Inhibition of cyclosporin A-induced gingival overgrowth by azithromycin through phagocytosis: an in vivo and in vitro study. J Periodontol. 2004; 75 (3): 380-387.

17. Mavrogiannis M, Ellis JS, Seymour RA, Thomason JM. The efficacy of three different surgical techniques in the management of drug- induced gingival overgrowth. J Clin Periodontol. 2006; 33 (9): 677-682.

18. Wu S, Applewhite AJ, Niezgoda J, Snyder R, Shah J, Cullen B et al. Oxidized regenerated cellulose/collagen dressings: review of evidence and recommendations. Adv Skin Wound Care. 2017; 30 (11S Suppl 1): S1-S18.

19. Chowdhry SA. Use of oxidized regenerated cellulose (ORC)/ collagen/silver-ORC dressings to help manage skin graft donor site wounds. JPRAS Open. 2019; 22: 33-40.

20. Kim SH, Kim SH, Yoon HS, Kim HK, Kim KS. Efficacy of oxidized regenerated cellulose, SurgiGuard $\AA$, in porcine surgery. Yonsei Med J. 2017; 58 (1): 195-205.

21. Frantz VK. Absorbable cotton, paper and gauze: (oxidized cellulose). Ann Surg. 1943; 118 (1): 116-126.

22. Schonauer C, Tessitore E, Barbagallo G, Albanese V, Moraci A. The use of local agents: bone wax, gelatin, collagen, oxidized cellulose. Eur Spine J. 2004; 13 Suppl 1: S89-S96.

23. Amaíz Flores AJ. La electrocirugía en la Odontología actual. Odontología Vital. 2018; (28): 91-101.

Conflicto de intereses: No existe conflicto de intereses. Financiamiento: Ninguno.

Correspondencia:

Luis Eduardo Poblano-Vázquez

E-mail: e_poblanovaz@hotmail.com 